\title{
A LASER METROLOGY/VIEWING SYSTEM FOR ITER IN-VESSEL INSPECTION ${ }^{*}$ CONF-97/065-
}

\section{P. T. Spampinato, R. E. Barry, J. B. Chesser, M. M. Menon,}

M. A. Dagher, ${ }^{\dagger}$ A. Slotwinski ${ }^{\ddagger}$

Oak Ridge National Laboratory

P.O. Box 2008

Oak Ridge, TN 37831-6304

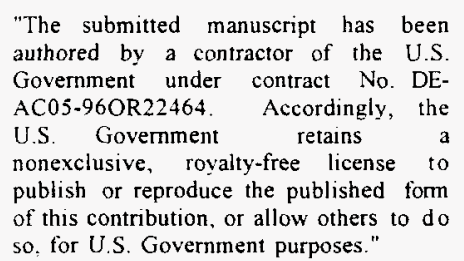

To be published in the Proceedings of the 1997 Symposium on Fusion Engineering,

San Diego, California, October 6-10, 1997

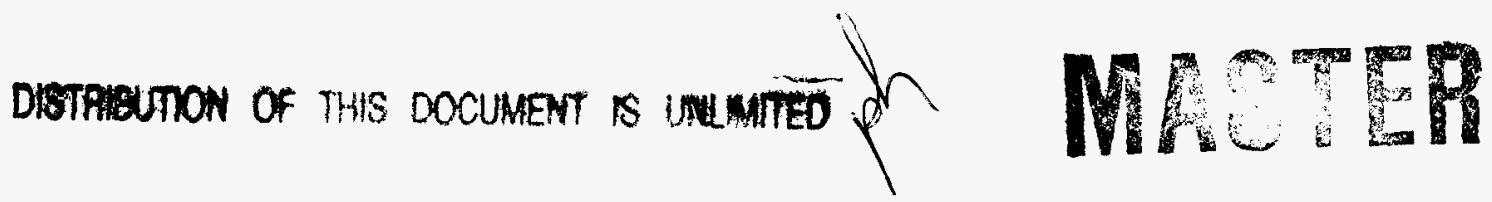

\footnotetext{
- Research sponsored by the Office of Fusion Energy Science, U.S. Department of Energy, under contract DE-AC05-96OR22464 with Oak Ridge National Laboratory, managed by Lockheed Martin Energy Research Corporation.

+Boeing Rocketdyne Division, 6633 Canoga Avenue. Canoga Park. CA 91309.

${ }^{+}$Coleman Research Corporation, 655I Loisdale Court, Suite 800. Springfield. VA 22150.
} 


\section{DISCLAIMER}

This report was prepared as an account of work sponsored by an agency of the United States Government. Neither the United States Government nor any agency thereof, nor any of their employees, make any warranty, express or implied, or assumes any legal liability or responsibility for the accuracy, completeness, or usefulness of any information, apparatus, product, or process disclosed, or represents that its use would not infringe privately owned rights. Reference herein to any specific commercial product, process, or service by trade name, trademark, manufacturer, or otherwise does not necessarily constitute or imply its endorsement, recommendation, or favoring by the United States Government or any agency thereof. The views and opinions of authors expressed herein do not necessarily state or reflect those of the United States Government or any agency thereof. 


\title{
A Laser Metrology/Viewing System for ITER In-Vessel Inspection*
}

\author{
P. T. Spampinato, ${ }^{a}$ R. E. Barry, ${ }^{a}$ J. B. Chesser, ${ }^{a}$ M. M. Menon ${ }^{a}$ \\ M. A. Dagher, A. Slotwinski ${ }^{\circ}$ \\ Oak Ridge National Laboratory P.O., Box 2008, Oak Ridge, TN 37831 \\ 'Boeing Rocketdyne Division, 6633 Canoga Avenue, Canoga Park, CA 91309 \\ "Coleman Research Corporation, 6551 Loisdale Court, Suite 800, Springfield. VA 22150
}

\begin{abstract}
This paper identifies the requirements for a remotely operated precision laser ranging system for the International Thermonuclear Experimental Reactor. The inspection system is used for metrology and viewing, and must be capable of achieving submillimeter accuracy and operation in a reactor vessel that has high gamma radiation, high vacuum, elevated temperature, and magnetic field levels. A coherent, frequency modulated laser radar system is under development to meet these requirements. The metrology/viewing sensor consists of a compact laser-optic module linked through fiberoptics to the laser source and imaging units, located outside the harsh environment. The deployment mechanism is a remotely operated telescopic mast. Gamma irradiation up to $10^{7}$ Gy was conducted on critical sensor components with no significant impact to data transmission, and analysis indicates that critical sensor components can operate in a magnetic field with certain design modifications. Plans for testing key components in a magnetic field are underway.
\end{abstract}

\section{INTRODUCTION}

The International Thermonuclear Experimental Reactor (ITER) is a fusion device planned to be built early in the next century. The performance and survival of plasma-facing components (PFCs) located in the reactor vacuum vessel will depend on precise alignment and positioning with respect to the plasma edge. A remotely operated, three-dimensional metrology system is being developed to periodically verify the surface condition of in-vessel components and to measure surface erosion. It consists of a laser-optic scanner deployed via a remotely operated telescoping mast. This metrology system has two basic functions: frequent inspection to establish the dimensional status of in-vessel components, and extensive checking of plasma-facing surfaces during scheduled maintenance shutdowns.

\section{DESIGN REQUIREMENTS}

The interior surface area of ITER is approximately $1500 \mathrm{~m}^{2}$. In order to scan all divertor high heat flux surfaces and achieve acceptable mapping times, 10 metrology systems will be required. Each system must be capable of acquiring in-vessel dimensional data to submillimeter accuracy under harsh environmental and radiological conditions. They also must withstand gamma radiation levels al $3 \times 10^{4} \mathrm{~Gy} / \mathrm{h}$ while operating in a $200^{\circ} \mathrm{C}$ environment in vacuum conditions. In addition, the systems must be deployed into a constant magnetic field of 6.2 Tesla. The metrology system must also function during scheduled maintenance activities, when the vessel will be at atmospheric pressure.

\section{DEPLOYMENT SYSTEM}

The viewing/metrology deployment system is designed to transport the laser scanner into the vacuum vessel to survey damage of PFC surfaces. The deployment system and its supporting structure were originally located on top of the bio-shield at $\mathrm{R}=7450 \mathrm{~mm}$ from the reactor center. A series of openings in the bio-shield, cryostat, and vacuum vessel allow the telescopic mast to be extended

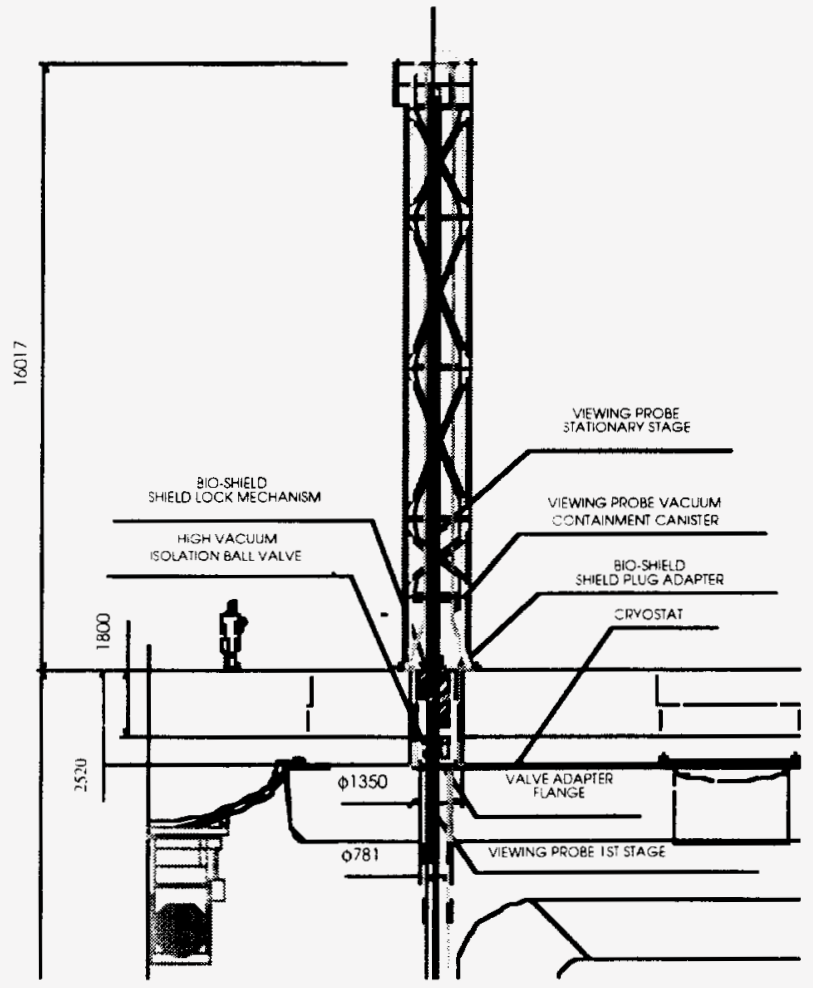

Fig. 1. Deployment mast mounted above the bio-shield

\footnotetext{
* Research sponsored by the Otfice of Fusion Energy Science. U.S. Department of Energy, under contract DE-ACO5-96OR22464 with Oak Ridge National Laboratory. Inanaged by Lockheed Martin Energy Research Corporation.
} 
down to the region of the divertor. Figure 1 provides a cutaway view of the deployment system mounted above the bio-shield. A recent change in design philosophy requires that the deployment system be located below the bio-shield.

During reactor operation, the mast will be stowed inside a vacuum container and isolated from the vacuum vessel by means of a shield plug mechanism at the vessel port, a vacuum isolation valve at the port opening and at the cryostat, and another shielding mechanism at the bio-shield. All of these components are designed to open and close in a specific sequence to preclude neutron streaming and to control potential contamination.

\section{A. Support Structure}

The deployment system support structure is approximately $18 \mathrm{~m}$ tall and is designed to provide structural/seismic support for the telescopic mast and the double containment vessel. It is mounted directly to the bio-shield. This interface consists of a set of mating flanges, one mounted on the structure and the other mounted at the bio-shield opening. In order to achieve the vacuum isolation required during mast deployment, a mechanical seal system is used between the two flanges. A set of bolts connects the two flanges and provides the necessary support for the deployment system.

\section{B. Containment Vessels}

Two containment vessels house the telescopic mast and provide the required double containment. The inner vessel is designed for ultra-high vacuum atmosphere and provides the support points for mounting the telescopic mast and its components. Support points transfer loads from the mast to the support structure. Vacuum-rated feedthrough openings are used to route all necessary cables and coolant lines into the telescopic mast. A double-door isolation mechanism is mounted at the lower end of the inner vessel at the bio-shield level. The double-door arrangement is designed to prevent the spread of contamination when the deployment system is removed.

\section{Telescopic Mast}

The telescopic mast delivers the laser sensor into the tokamak during viewing and inspection campaigns. It will position the sensor at various elevations and provide all necessary power and coolant to run the sensor head. which is mounted at the tip of the deployment mast-second stage. The telescopic mast is composed of three circular concentric tubes. The outer tube is stationary and supports the two movable tubes, as well as the drive unit and the cable reels mounted at the top. Tracks and guide rollers are installed between the tubes to provide the necessary alignment and to guide the mast during deployment. The mast tubes are driven by two sets of redundant cables, which are reeled and unreeled by the drive.

\section{Shielding Mechanisms}

During reactor operation, port openings must be shielded to prevent neutron streaming and minimize damage to the magnets. Primary shielding is required at the vacuum vessel ports just above the shield blanket. This is achieved by using a shield lock mechanism composed of a series of stationary and rotating disks. During mast deployment, the moving disks rotate to align the openings and provide access for the mast to pass through the shield plug assembly. This shielding mechanism is designed to provide an equivalent shielding thickness of $1 \mathrm{~m}$ at all times. Additional shielding is also required at the bio-shield. A similar shield mechanism is used at this level, except it has a larger opening to accommodate the first stage of the telescopic tube.

\section{LASER RADAR METROLOGY SYSTEM}

\section{A. Background}

The metrology system is a high-precision frequency modulated (FM) coherent laser radar (CLR) ranging device. The range is measured by the shift in frequency of the FM laser beam reflected off the target. The laser frequency is modulated linearly by means of a special waveform, and the range accuracy is determined by the linearity of the frequency modulation over the counting interval.

A lightwave signal from the laser source sweeps linearly in frequency with time. Lightwave signals that have traveled to the target or through the local oscillator fiberoptic loop will have a delay resulting in a constant beat frequency when the two signals are mixed at the detector. This beat frequency is proportional to the path-length difference between the target path and the local oscillator path.

The laser metrology system is being designed to interface with the vertical deployment mast; fiberoptic connections, cooling lines, and power signals will be carried through the deployment system to the sensor interface. The sensor package will be completely self-contained, with all umbilical connections vacuum rated. The connection of the sensor package to the deployment mast will be designed as a hands-on operation for a rad-worker wearing anti-contamination clothing.

The sensor is based on a design proposed by Coleman Research Corporation [1]. Coleman's previous work in this area yielded a precision laser radar system with a wide field of view. ixtremely high range resolution. and a high degree of programmability. ITER requirements. however, demand that a different sensor head and additional components be added to the current system to meet the ITER operational scenarios. Figure 2 shows the Coleman equipment measuring a simulated PFC. 
An additional challenge is to develop the metrology system for operation in the magnetic tieid inside the torus. The sensor is to be used with the toroidal field coils energized. This affects the sensor drive and positioning components. Operation in a magnetic field requires that electric drive motors must be eliminated. or placed remotely from the sensor, and located in the first stage of the telescopic mast. Position encoders must not be electromagnetically based. or they must also be placed remotely from the sensor. Alternative components under consideration include piezo-actuators, optical encoders, and capacitive encoders.

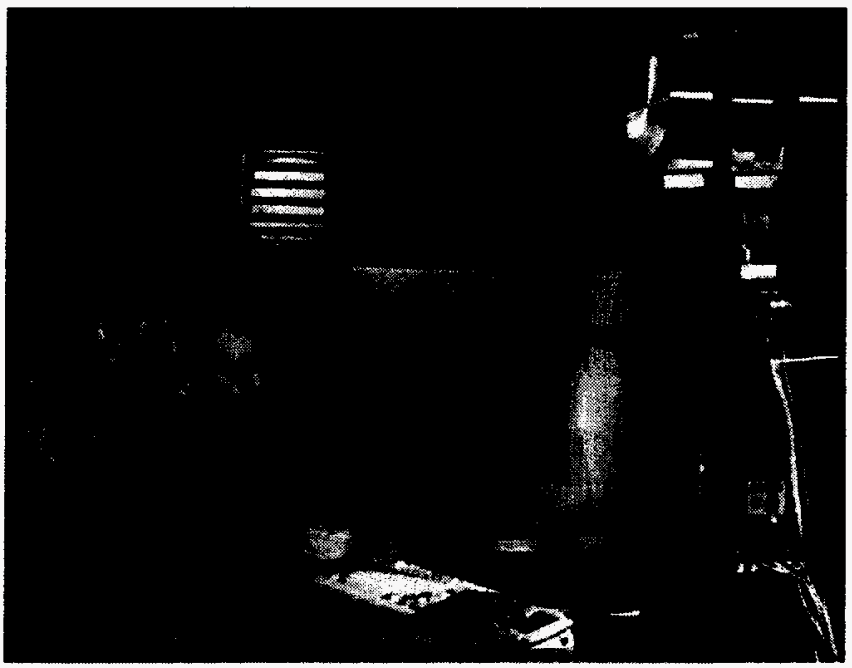

Fig. 2. CLR measuring a simulated tokamak component.

\section{B. Operation}

The sensor will be capable of 20-meter range measurements. with a range precision of approximately 100 micrometers. The design concept supports a field of view that is $\pm 185^{\circ}$ in azimuth and $100^{\circ}$ in elevation. This is accomplished by sweeping a $7^{\circ}$ azimuth $\times 100^{\circ}$ elevation scan around the vertical axis of the sensor mast. Sensor resolution will be programmable over a range of approximately I point per $1 \mathrm{~mm}^{2}$ millimeter square to approximately 1 point per $\mathrm{cm}^{2}$.

The sensor module is attached to the remotely operated deployment mast. It steers the rangefinder laser beam, focuses the beam, and provides feedback on beam direction. The first element of the module is the rotary joint to point the beam in the azimuthal direction and provide coarse positioning. Fine sweeping is provided by an acousto-optic (AO) device within the sensor. Below the rotary joint is the main sensor body, which houses the focusing optics. the $\mathrm{AO}$ cell for beam steering, and the nodding mirror.

A polarization maintaining (PM) optical fiber carries the beam to the sensor head. The beam is focused and directed into the $\mathrm{AO}$ crystal. where it is swept from side to side. The heam then passes through a wedge window and on to the nodding mirror. The wedge window is provided to angle the beam to the nodding mirror or to allow the beam to scan straight down.

\section{Radiation Effects on Sensor Components}

Very limited data are available on the compatibility of certain components of the sensor in the high radiation environment. The AO scanner and the PM fiber fall into this category. In the AO device, the effect of high gamma radiation on the $\mathrm{TeO}_{2}$ crystal was not known. Samples of the $\mathrm{TeO}_{2}$ crystal and the PM fiber were tested under high gamma radiation at the High Flux Isotope Reactor facility at Oak Ridge National Laboratory (ORNL). Two crystals were irradiated, one to $5.9 \times 10^{8} \mathrm{~Gy}$, the other to $1.4 \times 10^{7} \mathrm{~Gy}$. The fiber was irradiated to $1.4 \times 10^{7} \mathrm{~Gy}[2]$.

Although the radiation produced noticeable discoloration to the crystal (radiation-induced browning), there was no noticeable effect on the transmission characteristics of the crystal in the operating wavelength range $(-1500 \mathrm{~nm})$. Identical results were obtained for the second crystal. Therefore, it was concluded that the basic $\mathrm{TeO}_{2}$ crystal is sufficiently rad-hard for ITER application. Conclusions from the fiber irradiation were as follows: (1) degradation in laser transmission occurred with radiation dose, but the deterioration can be readily compensated by gain adjustments, and (2) although insufficient data were collected for a plot, the ratio of the two polarization components measured at two discrete intervals was constant, suggesting that the polarization did not change with irradiation. Future plans include irradiation of a complete $\mathrm{AO}$ device that will include the lithium niobate electro-acoustic transducer.

\section{Magnetic Field Effects}

Preliminary estimates have been made regarding the effects of operating the device in a steady magnetic field of $6.2 \mathrm{~T}$. The direction of the field is toroidal and therefore orthogonal to the direction of probe deployment. The force on the boom during insertion is in the axial direction (approximately 100 $\mathrm{N}$ for an insertion velocity of $1 \mathrm{~m} / \mathrm{min}$ ) and can be further reduced by reducing the insertion velocity. The $\mathrm{J} \times \mathrm{B}$ forces acting on the electrical leads of the AO device do not appear to be problematic. The primary problem is to design a remote actuating device for adjusting and monitoring the mirror position that can operate reliably. The operation of the AO device, two types of position encoders, and a piezo-actuator will be tested in the High-Field Magnet Test Facility at ORNL.

\section{SENSOR MEASUREMENTS}

Several different materials are under consideration for PFCs. Because the range accuracy of most laser-based ranging systems is highly dependent on the surface material type, the different materials for ITER were analyzed for their effect on range measurements. Beryllium, tungsten, and a carbon fiber 
composite (graphite) were chosen for analysis. Each material was placed in a rotary stage and moved through a range of angles between 0 and 90 degrees, with approximately 100 measurements made at each angle. The standard deviation of the highest quality range measurements was found to be acceptable [3].

Further ranging tests were made with the Coleman system on a heat-damaged beryllium tile from the ORNL ISX-B fusion reactor. The tile used is shown in Fig. 3. The damage can be clearly seen at the apex of the tile's top surface. Using a scan pattern of $100 \times 100$ points and measuring at a distance of $4.6 \mathrm{~m}$, a surface mesh of the range measurement data yields the plot shown in Fig. 4. Additional testing will be done to assess range precision as a function of angle of incidence.

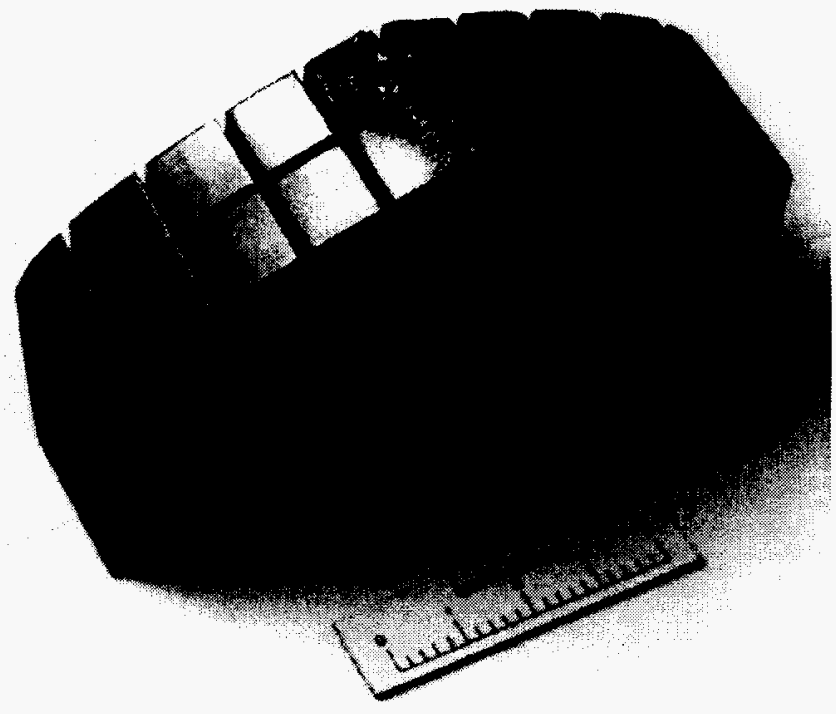

Fig. 3. Plasma damaged tile.

\section{CONCLUSIONS}

A remotely operated deployment mast and an FM CLR system are being developed for remote metrology of plasma facing surfaces in ITER. The work to date hows that the system is capable of providing both fualitative and quantitative information regarding plasma-facing surface conditions. Encouraging results were obtained from testing key components for radiation tolerance. and preliminary analysis indicates that operating in a magnetic field is possible. Although the device is being developed for metrology and inspection of internal fusion reactor components, it can be applied to a variety of other applications involving precision measurements and viewing of components in areas not accessible to personnel.

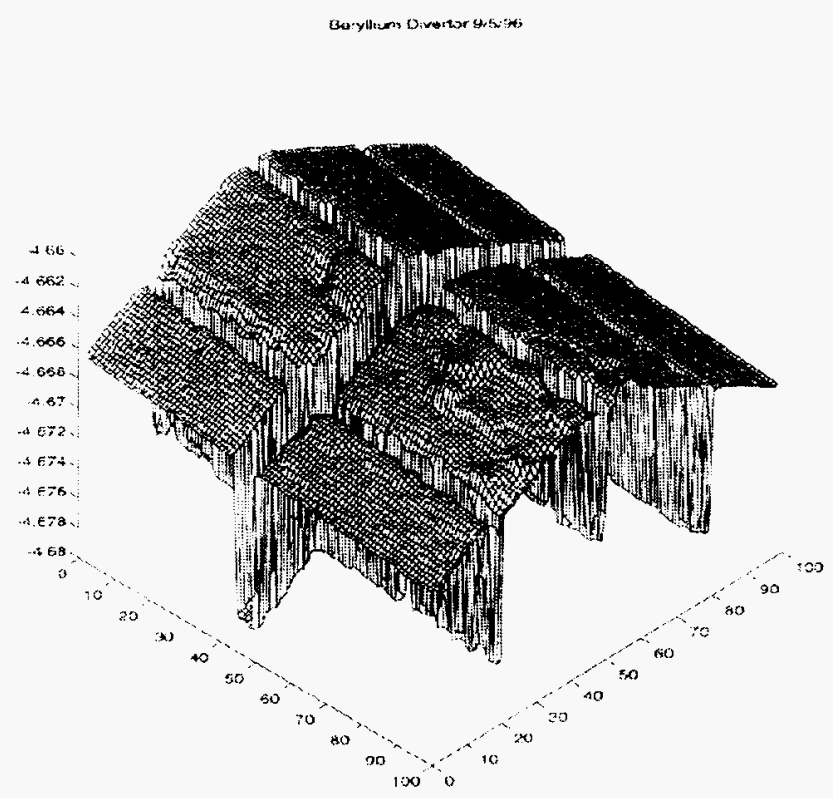

Fig. 4. Range measurement data from the Be tile.

\section{REFERENCES}

[1] R. E. Barry, M. M. Menon, and A. Slotwinski, "A Three Dimensional Remote Metrology System for the International Thermonuclear Experimental Reactor," Proc. American Nuclear Society Winter Meeting, San Francisco, California, 1995.

[2] M. M. Menon, R. E. Barry, P. T. Spampinato, and A. Slotwinski, "A Remotely Operable Sensor for Precision Mapping Using Coherent FM Laser Radar," Proc. 126th TMS Annual Meeting, Orlando, Florida, 1997.

[3] P. Spampinato, R. E. Barry, M. M. Menon, M. A. Dagher, and A. Slotwinski, A Laser Scanning System for Metrology and Viewing in ITER, Proc. Sixth International Symposium on Robotics and Manufacturing, Montpellier, France, 1996. 
,

(n)
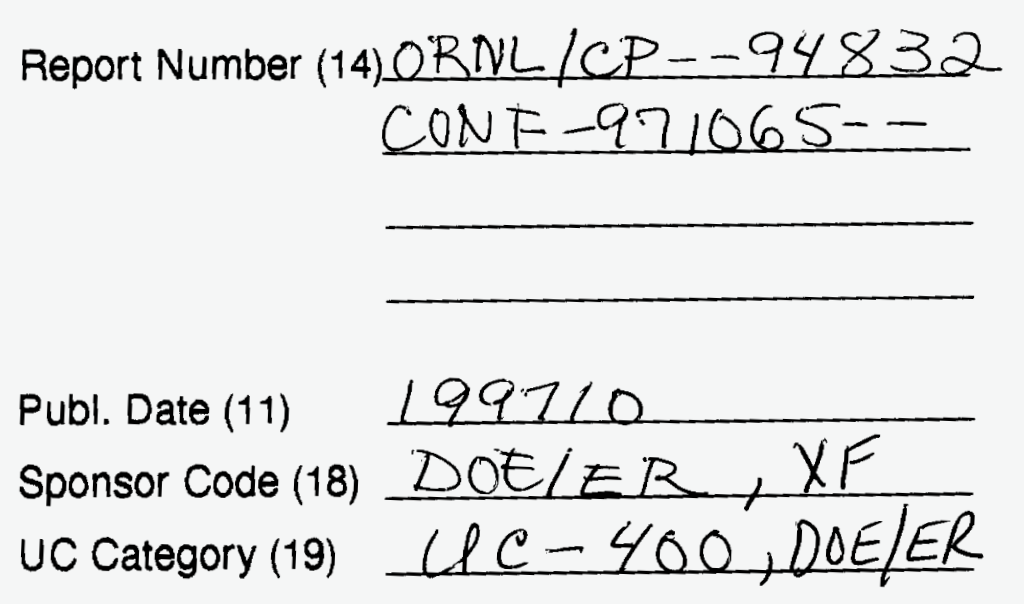

DOE 